\title{
A COMPUTATIONAL ALGORITHM FOR MINIMAX PROBLEMS WITH CONSTRAINTS
}

\author{
HITOSHI SASAI, Yokohama National C'niversity
}

(Received May 7, 1976; Revised November 2, 1976)

\begin{abstract}
In this paper we propose an interior penalty method to solve a minimax problem with inequality and equality constraints.

The constrained minimax problem is replaced by a sequence of unconstrained approximate minimax problems.

It is shown that the sequence of solutions of unconstrained approximation problems exists and converges to the solution of original constrained minimax problem.
\end{abstract}

\section{INTRODUCTION}

The minimax problem with side constraints is interesting from a theoretical and practical standpoint, but is difficult to solve directly. Therefore it is necessary to obtain a numerical solution by approximating the original problem in computationally feasible manner.

On the other hand it is well known that the penalty function approach is powerful for solving nonlinear programming problems.

The idea originates in R. Courant $|1|$ and its further development is due to A. V. Fiacco and G. P. McCormic |2|, |3|.

The penalty function method cannot be directly applied for the minimax problem. Its approach, however, very useful for our discussion by introducing a new convex-concave penalty funcricin.

We use the penalty function technique in order to solve the minimax problem. As a result the constraned minimax problem is reduced to the one solving a sequence of unconstrained approximations.

This type of approximation has been discussed by the present author $|4|$ in the restricted case where the constraints are simply inequalities. 
We extend this method to the case where the constraints are a mixture of both equalities and inequalities by proposing a new type of penalty function.

In this paper we are mainly concerened with conditions of convergence of approximation problems, because the existence of solutions of approximation problems can be easily proved by a similar techniques as [4].

\section{STATEMENT OF PROBLEM}

Let $*$ and $\mathrm{u}$ be finite dimensional Euclidean spaces.

Now we consider the functions:

$\mathrm{f}(\mathrm{x}, \mathrm{u}) ; * x \mathrm{u} \rightarrow \mathrm{R}^{1}$

$\mathrm{g}_{\mathrm{i}}(\mathrm{x}), \widetilde{\mathrm{g}}_{\mathrm{j}}(\mathrm{x}) ; * \rightarrow \mathrm{R}^{1}$, where $\mathrm{i}=1, \ldots . . \mathrm{I}$ and $\mathrm{j}=1, \ldots . . \mathrm{J}$,

$\mathrm{h}_{\mathrm{k}}(\mathrm{u}), \widetilde{\mathrm{h}}_{\ell}(\mathrm{u}) ; \mathrm{u} \rightarrow \mathrm{R}^{1}$, where $\mathrm{k}=1, \ldots . ., \mathrm{K}$ and $\ell=1, \ldots ., \mathrm{L}$.

Let us define the constraint sets as follow:

$$
\begin{aligned}
& X_{1}=\left[x: g_{i}(x) \geqslant 0, i=1, \ldots ., I\right], X_{1}^{0}=\left[x: g_{i}(x)>0, i=1, \ldots ., I\right], \\
& X_{2}=\left[x: \widetilde{g}_{j}(x)=0, j=1, \ldots ., J\right], \\
& U_{1}=\left[u: h_{k}(u) \geqslant 0, k=1, \ldots ., K\right], U_{1}^{0}=\left[u: h_{k}(x)>0, k=1, \ldots ., K\right], \\
& U_{2}=\left[u: \widetilde{h}_{\ell}(u)=0, \ell=1, \ldots ., L\right] .
\end{aligned}
$$

The minimax problem under consideration is the following one.

Original Problem $(O)$ "Find a saddle point of $f(x, u)$ with respect to $\left(X_{1} \cap X_{2}\right) \times\left(U_{1} \cap U_{2}\right)$, i.e., a point $(\hat{x}, \hat{u}) \in\left(X_{1} \cap X_{2}\right) \times\left(U_{1} \cap U_{2}\right)$ such that

$f(\hat{x}, u) \leqslant f(\hat{x}, \hat{u}) \leqslant f(x, \hat{u})$

for any $\mathrm{x} \in \mathrm{X}_{1} \cap \mathrm{X}_{2}$ and $\mathrm{u} \in \mathrm{U}_{1} \cap \mathrm{U}_{2} . "$

An attempt to solve Prob. (O) by applying an penalty method would result in the sequence of following problems.

Approximation Problem (A) "Find a saddle point of $P\left(x, u, r_{n}\right)$ with respect to $X_{1}^{0} \times U_{1}^{0}$ for each $r_{n}>0$, where

$$
\begin{aligned}
P\left(x, u, r_{n}\right)= & f(x, u)+\sum_{i=1}^{I} \frac{r_{n}}{g_{i}(x)}+\sum_{j=1}^{J} \frac{\widetilde{g}_{j}^{2}(x)}{r_{n}} \\
& -\sum_{k=1}^{K} \frac{r_{n}}{h_{k}(u)}-\sum_{\ell=1}^{L} \frac{\widetilde{h}_{\ell}^{2}(u)}{r_{n}}
\end{aligned}
$$

and $r_{n}$ is a strictly monotonic decreazing sequence tending to zero."

Now we can propose the following procedure to solve Prob. (O).

As a starting point for the process determine $\mathrm{x}_{1} \in \mathrm{X}_{1}^{0}$. For the fixed $\mathrm{x}_{1}$, find a point $\mathrm{u}_{1}$ which maximizes $P\left(x_{1}, u, r_{1}\right)$ in the feasible domain $U_{1}^{0}$. For the fixed $u_{1}$, find a point $x_{2}$ which minimizes $P\left(x, u_{1}, r_{1}\right)$ in $X_{1}^{0}$. Continuing in this manner, we can find a saddle point $\left(x_{r_{2}}, u_{r_{1}}\right)$ of $P\left(x, u, r_{1}\right)$, if 
we put appropriate conditions to each function as stated below. Form the new function $\mathrm{P}\left(\mathrm{x}, \mathrm{u}, \mathrm{r}_{2}\right)$, where $r_{2}<r_{1}$. Starting now from $x_{r_{1}}$, determine a saddle point of $P\left(x, u, r_{2}\right)$ by the similar way . Continuing in this manner, a sequence of points $\left(x_{r_{n}}, u_{r_{n}}\right)$ are generated.

If we can assure that this sequence converges to a saddle point of Prob. $(\mathrm{O})$, new computational algorithm is established.

\section{THEOREMS}

We assume that the following conditions are imposed on our discussions.

$\left(c_{1}\right): f(x, u)$ is a convex-concave function and continuous on $* x u$.

$\left(c_{2}\right): g_{i}(x)$ and $h_{k}(u)$ are concave and continuous, where $\mathrm{i}=1, \ldots \ldots, I$ and $k=1, \ldots ., \mathrm{K}$.

$\left(c_{3}\right): \quad \widetilde{g}_{j}^{2}(x)$ and $-\widetilde{h}_{\ell}^{2}(u)$ are convex, and $\widetilde{g}_{j}(x)$ and $\widetilde{h}_{\ell}(x)$ are continuous, where $j=1, \ldots ., J$ and $\ell=1, \ldots . ., \mathrm{L}$.

$\left(c_{4}\right): \quad X_{1}$ and $U_{1}$ are bounded respectively, and $X_{1}^{0} \cap X_{2} \neq \phi U_{1}^{0} \cap U_{2} \neq \phi$.

Remark. If $\left(c_{2}\right),\left(c_{3}\right)$ and $\left(c_{4}\right)$ are satisfied, it is easy to show that $\overline{X_{1}^{0} \cap X_{2}}=X_{1} \cap X_{2}$ and $\overline{\mathrm{U}_{1}^{0} \cap \mathrm{U}_{2}}=\mathrm{U}_{1} \cap \mathrm{U}_{2}$ by using the concavity, convexity and continuity of each function and $\left(c_{4}\right)$, where $\bar{A}$ denotes the closure of a set $A$ in an Euclidean space [6].

THEOREM 1. Let the conditions $\left(c_{1}\right),\left(c_{2}\right),\left(c_{3}\right)$ and $\left(c_{4}\right)$ be satisfied. Suppose that there exists a saddle point of Prob. (A) for each $r_{n}$, which we denote by $\left(x_{r_{n}}, u_{r_{n}}\right)$. Then there exists a subsequence of $\left(\mathrm{x}_{\mathrm{r}_{\mathrm{n}}}, \mathrm{u}_{\mathrm{r}_{\mathrm{n}}}\right)$ converging to a saddle point of Prob. (O).

PROOF. Since $X_{1}$ and $U_{1}$ are bounded and closed by $\left(c_{2}\right),\left(c_{3}\right)$ and $\left(c_{4}\right)$, there exists a subsequence of $\left(x_{r_{n}}, u_{r_{n}}\right)$ converging to an $(\hat{x}, \hat{u}) \in X_{1} \times U_{1}$. We use the same symbol $\left(x_{r_{n}}, u_{r_{n}}\right)$ for a subsequence.

We shall prove that $(\hat{x}, \hat{u})$ is a saddle point of Prob. $(O)$. Since $\left(x_{r_{n}}, u_{r_{n}}\right)$ is a saddle point of Prob. (A),

$$
P\left(x_{r_{n}}, u, r_{n}\right) \leqslant P\left(x_{r_{n}}, u_{r_{n}}, r_{n}\right) \leqslant P\left(x, u_{r_{n}}, r_{n}\right)
$$

for any $\mathrm{x} \in \mathrm{X}_{1}^{0}$ and $\mathrm{u} \in \mathrm{U}_{1}^{0}$. By (1), for any $\mathrm{x} \in \mathrm{X}_{1}^{0}$

$$
\begin{aligned}
& f\left(x_{r_{n}}, u_{r_{n}}\right) \leqslant f\left(x_{r_{n}}, u_{r_{n}}\right)+\Sigma \frac{I}{g_{i}\left(x_{r_{n}}\right)}+r_{\Sigma} \frac{\widetilde{g}_{j}^{2}\left(x_{r_{n}}\right)}{r_{n}} \\
& \leqslant f\left(x, u_{r_{n}}\right)+\Sigma \frac{I}{\Sigma} \frac{r_{n}}{g_{i}(x)}+\Sigma \frac{J}{g_{j}^{2}(x)} \frac{r_{n}}{r_{n}}
\end{aligned}
$$

Hence, for any $\mathrm{x}_{0} \in \mathrm{X}_{1}^{0} \cap \mathrm{X}_{2}$ which exists by $\left(c_{4}\right)$, 


$$
\begin{aligned}
& f\left(x_{r_{n}}, u_{r_{n}}\right) \leqslant f\left(x_{r_{n}}, u_{r_{n}}\right)+\Sigma \frac{I}{g_{i}\left(x_{r_{n}}\right)}+\sum \frac{r_{n}}{\tilde{g}_{j}\left(x_{r_{n}}\right)} \\
& \leqslant f\left(x_{0}, u_{r_{n}}\right)+\Sigma \frac{I}{g_{i}\left(x_{0}\right)} .
\end{aligned}
$$

Thus, we have the following inequality by $\left(c_{1}\right)$ and $\left(c_{3}\right)$.

$$
\mathrm{f}(\hat{\mathrm{x}}, \hat{\mathrm{u}}) \leqslant \mathrm{f}\left(\mathrm{x}_{0}, \hat{\mathrm{u}}\right) \quad \text { for any } \mathrm{x}_{0} \in \mathrm{X}_{1}^{0} \cap \mathrm{X}_{2} .
$$

Now we can show $\hat{x} \in X_{2}$. Since $\left(x_{r_{n}}, u_{r_{n}}\right) \in X_{1}^{0} \times U_{1}^{0}, X_{1} \times U_{1}$ is compact and $f(x, u)$ is continuous on $* x \mathrm{u}$ by $\left(c_{1}\right), f\left(x_{r_{n}}, u_{r_{n}}\right)$ is uniformly bounded. Therefore, by the inequality of (3), ${ }_{\Sigma}^{J} \widetilde{g}_{j}^{2}\left(x_{r_{n}}\right) / r_{n}$ becomes to be uniformly bounded, and so $\Sigma \widetilde{g}_{j}^{2}\left(x_{r_{n}}\right) \rightarrow 0$. Consequently $\lim \Sigma \widetilde{g}_{j}^{2}\left(x_{r_{n}}\right)$ $=\Sigma \widetilde{g}_{j}^{2}(\hat{x})=0$ because each $\widetilde{g}_{j}$ is continuous. This facts mean $\hat{x} \in X_{2}$.

By the similar technique we have

$$
\mathrm{f}(\hat{\mathrm{x}}, \hat{\mathrm{u}}) \geqslant \mathrm{f}\left(\hat{\mathrm{x}}, \mathrm{u}_{0}\right) \text { for any } \mathrm{u}_{0} \in \mathrm{U}_{1}^{0} \cap \mathrm{U}_{2} \text {, }
$$

and $\hat{\mathrm{u}} \in \mathrm{U}_{2}$.

Since $\overline{X_{1}^{0} \cap X_{2}}=X_{1} \cap X_{2}$ and $\overline{U_{1}^{0} \cap U_{2}}=U_{1} \cap U_{2}$ by Remark, for any feasible $(x, u)$ there exists a sequence $\left\{\left(x_{n}, u_{n}\right)\right\} \subset\left(X_{1}^{0} \cap X_{2}\right) \times\left(U_{1}^{0} \cap U_{2}\right)$ which conveges to $(x, u)$.

On the other hand, by (4) and (5), we have

$$
f\left(\hat{x}, u_{n}\right) \leqslant f(\hat{x}, \hat{u}) \leqslant f\left(x_{n}, \hat{u}\right) .
$$

f being continuous, we can conclude:

$$
\mathrm{f}(\hat{\mathrm{x}}, \mathrm{u}) \leqslant f(\hat{\mathrm{x}}, \hat{\mathrm{u}}) \leqslant \mathrm{f}(\mathrm{x}, \hat{\mathrm{u}})
$$

for any $\mathrm{x} \in \mathrm{X}_{1} \cap \mathrm{X}_{2}$ and $\mathrm{u} \in \mathrm{U}_{1} \cap \mathrm{U}_{2}$, and $\hat{\mathrm{x}} \in \mathrm{X}_{1} \cap \mathrm{X}_{2}$, $\mathrm{u} \in \mathrm{U}_{1} \cap \mathrm{U}_{2}$.

Remark. Under the cond. $\left(c_{1}\right) \sim\left(c_{4}\right)$ the existence of a solution of Prob. $(O)$ is assured by another technique [5]. But we can see by this theorem that the existence of solutions of Prob. (A) implies the existence of a solution of Prob. (O).

COROLLARY 1. Let the conditions stated in Theorem 1 be satisfied. If a saddle point of Prob. (O) denoted by $(\hat{x}, \hat{u})$ is unique, $\left(x_{r_{n}}, u_{r_{n}}\right)$ converges to $(\hat{x}, \hat{u})$.

PROOF: This corollary is clear because any subsequence of $\left(\mathrm{x}_{r_{n}}, u_{r_{n}}\right)$ has also its subsequence converging to $(\hat{\mathrm{x}}, \hat{\mathrm{u}})$.

Concerning the existence of solutions of Prob. (A), we can use the same technique as that in [4]. The function $P\left(x, u, r_{n}\right)$ can be modified such that its modification is a convex-concave extension under $\left(c_{1}\right) \sim\left(c_{4}\right)$. After such a modification Moreau's theorem [4] is directly applied and we have the following theorem. 
THEOREM 2. Let the conditions $\left(c_{1}\right),\left(c_{2}\right),\left(c_{3}\right)$ and $\left(c_{4}\right)$ be satisfied. Then there exists a saddle point of $\mathrm{P}\left(\mathrm{x}, \mathrm{u}, \mathrm{r}_{\mathrm{n}}\right)$ with respect to $\mathrm{X}_{1}^{0} \times \mathrm{U}_{1}^{0}$.

In what follows, we denote the saddle point of Prob. (A) for each $r_{n}$ by $\left(x_{r_{n}}, u_{r_{n}}\right)$.

COROLLARY: If $f(x, u), g_{i}(x), \widetilde{g}_{j}(x), h_{k}(u)$ and $\widetilde{h}_{\ell}(u)$ are all differentiable in each variable, then

$$
\begin{aligned}
& \left.\nabla_{\mathrm{x}} \mathrm{P}\left(\mathrm{x}, \mathrm{u}_{\mathrm{r}_{\mathrm{n}}}, \mathrm{r}_{\mathrm{n}}\right)\right|_{\mathrm{x}=\mathrm{x}_{\mathrm{r}_{\mathrm{n}}}}=0 \\
& \nabla \mathrm{u} P\left(\mathrm{x}_{\mathrm{r}_{\mathrm{n}}}, \mathrm{u}, \mathrm{r}_{\mathrm{n}}\right) \mid \mathrm{u}=\mathrm{u}_{\mathrm{r}_{\mathrm{n}}}=0 \text {, }
\end{aligned}
$$

where $\nabla \mathrm{x}$ and $\nabla \mathrm{u}$ are the gradients of $\mathrm{P}\left(\mathrm{x}, \mathrm{u}, \mathrm{r}_{\mathrm{n}}\right)$ in $\mathrm{x}$ and $\mathrm{u}$, respectively.

PROOF: This corollary is clear because $\left(\mathrm{x}_{\mathrm{r}_{n}}, \mathrm{u}_{\mathrm{r}_{n}}\right) \in \mathrm{X}_{1}^{0} \times \mathrm{U}_{1}^{0}$ and $\mathrm{X}_{1}^{0}, \mathrm{U}_{1}^{0}$ are interior sets of $\mathrm{X}_{1}$ and $\mathrm{U}_{1}$, respectively.

\section{CONCLUSION}

In this paper we proposed an interior penalty method to solve a minimax problem with inequality and equality constraints.

Prob. $(\mathrm{O})$ is the original constrained minimax problem and Prob. (A) is the unconstrained minimax problem.

The algorithm proposed is to solve the minimax problem of Prob. (A) for decreasing $r_{n}$. As a result, we can obtain a sequence of solutions of Prob. (A), which are all interior points of constrained sets.

We have shown that under stated conditions the sequence of solutions of Prob. (A) exists (Theorem 2) and converges to the solution of Prob. (O) (Corollary 1).

\section{REFERENCES}

[1] Courant, R.: Variational methods for the solution of problems of equilibrium and vibrations, Bull. Amer. Math. Soc., 49 (1943), pp. 1-23.

[2] Fiacce, A. V. and McCormic, G. P.: The sequential unconstrained minimization technique for nonlinear programming, a primal-dual method,Management Science, 10 (1964), pp. 360 $-366$.

[3] Fiacco, A. V. and McCormic, G. P.: Extensions of SUMT for nonlinear programming, Management Science. 12 (1969), pp. 816-828.

[4] Sasai, H.: An interior penalty method for minimax problems with constraints, SIAM J. on Control, 12 (1.974), pp. 643-649. 
[5] Rockafellor, R.T.: Convex Analysis,Princeton Univ. Press, Princeton, N.J., 1969.

[6] Yamamoto, Y., and Sasai, H.: On the Approximate Solutions of Optimal Control Problems with Inequality Constraints, Trans. SICE, 7 (1971), pp. 263-267. 